\title{
Diagnostics of metastasis: an increasing challenge with high clinical importance
}

\author{
Jörg Haier $^{1}$ (D)
}

Received: 22 May 2019 / Accepted: 4 June 2019 / Published online: 6 June 2019

(c) Springer Nature B.V. 2019

For decades, the clinically important step of diagnosing metastatic progression in cancer patients has been mainly limited to a yes/no-decision. This is still represented by the TNM classification, which only differentiates distant metastases as M0 or M1 with the addition of the involved host organs [1]. For a few cancer entities, such as appendix carcinoma C18.1 and colorectal carcinoma C18-20, subclassifications based on histological patterns and organ distribution can be used. By contrast, major advances in the clinical management of metastatic disease have been made, and can be expected to have enormous benefits for patients. These advances are mainly based on a better understanding of the development of metastatic disease, and on the characteristics of the processes that lie behind tumor spread. Modern treatment strategies for metastatic disease are more and more related to precisely defined molecular signatures, interactions with the tumor environment, and other cancer cell characteristics. There is a pressing need for clinical oncologists to include these developments in the standardized form of assessment and subsequent documentation of metastases. In this issue, Höppener et al. [2] present attempts to achieve such standards for the pathological examination of metastatic lesions. Their effort to describe and classify heterogeneity within colorectal metastases should trigger further discussion and evaluation of these proposed standardizations.

A thorough evaluation of metastases, such as representing the three-dimensional aspects of the lesions in standard pathological examinations as discussed in this paper, and the use of high-quality scientific approaches is badly needed. Healthcare economics puts a major pressure on pathologists to develop effective and efficient approaches that correctly and reproducibly describe growth patterns, in a manner that clinicians can use for their treatment decisions. Furthermore, a rapidly increasing number of therapeutic agents such as

Jörg Haier

Haier.joerg@mh-hannover.de

1 Hannover Medical School, Hannover, Germany small molecules, immune inhibitors and antibodies are finding potential application in the clinical treatment of progressive metastatic disease, which again demands more precise stratification and differentiation in the clinical-pathological description of such lesions. Technologies that need to be employed include molecular panels, neuronal networks, artificial intelligence learning etc. [3]. These parameters and the new diagnostic elements are still not represented in clinical standard evaluations, and cannot be incorporated into TNM usage. This increasingly results in a lack of comparability of metastatic disease descriptors, with subsequent problems in applying clinical evidence to the treatment of specific cancer cases. Clinicians, pathologists, statisticians and other specialists urgently need to implement reproducible, applicable and clinically relevant classifications that include these parameters and items. The TNM or ICD-O systems can provide a basis for these developments, but comparable evidence for molecular classification standards is a prerequisite.

In view of these observations, Clinical \& Experimental Metastasis wishes to encourage discussion of these issues, and wants to provide a platform for new developments and advances in this area. This is a task not only for clinical/ pathological investigation, but should also include and incorporate the current REMARK strategies for biomarker development [4].

\section{References}

1. Brierley JD, Gospodarowicz MK, Wittekind C (eds) (2017) TNM classification of malignant tumors, 8th edn. Wiley, Hoboken

2. Höppener DJ et al (2019) Histopathological growth patterns of colorectal liver metastasis exhibit little heterogeneity and can be determined with a high diagnostic accuracy. Clin Exp Metastasis. https://doi.org/10.1007/s10585-019-09975-0

3. Grewal JK, Tessier-Cloutier B, Jones M, Gakkhar S, Ma Y, Moore R, Mungall AJ, Zhao Y, Taylor MD, Gelmon K, Lim H, Renouf D, Laskin J, Marra M, Yip S, Jones SJM (2019) Application of a neural network whole transcriptome-based pan-cancer method for diagnosis of primary and metastatic cancers. JAMA Netw 
Open 2(4):e192597. https://doi.org/10.1001/jamanetworkopen .2019 .2597

4. Subcommittee of the NCI-EORTC Working Group on Cancer Diagnostics Reporting recommendations for tumour MARKer prognostic studies (REMARK). https://www.nature.com/artic les/6602678
Publisher's Note Springer Nature remains neutral with regard to jurisdictional claims in published maps and institutional affiliations. 